\title{
Minimum Tube Diameters for Steady Propagation of Gaseous Detonations
}

\author{
Received: 16 August 2013 / Revised: 23 December 2013 / Accepted 16 March 2014
}

\begin{abstract}
Recent experimental results on detonation limits are reported in this paper. A parametric study was carried out to determine the minimum tube diameters for steady detonation propagation in five different hydrocarbon fuel-oxygen combustible mixtures and in five polycarbonate test tube diameters ranging from $50 \mathrm{~mm}$ down to a small scale of $1.5 \mathrm{~mm}$. The wave propagation in the tube was monitored by optical fibers. By decreasing the initial pressure, hence the sensitivity of the mixture, the onset of limits is indicated by an abrupt drop in the steady detonation velocity after a short distance of travel. From the measured wave velocities inside the test tube, the critical pressure corresponding to the limit and the minimum tube diameters for the propagation of the detonation can be obtained. The present experimental results are in good agreement with previous studies and show that the measured minimum tube diameters can be reasonably estimated on the basis of the $\lambda / 3$ rule over a wide range of conditions, where $\lambda$ is the detonation cell size. These new data shall be useful for safety assessment in process industries and in developing and validating models for detonation limits.
\end{abstract}

Keywords Detonation - Limits - Minimum tube diameters · Safety

\section{PACS 47.40.Rs}

Y. Gao

School of Chemical Machinery

Dalian University of Technology

Dalian, 116024, China

J.H.S. Lee

Department of Mechanical Engineering, McGill University

Montréal, Québec, H3A 2K6, Canada

H.D. $\mathrm{Ng}$

Mechanical and Industrial Engineering, Concordia University

Montréal, Québec, H3G 1M8, Canada

Tel.: (514) 848-2424 x 3177

Fax: (514) 848-3175

E-mail: hoing@encs.concordia.ca

\section{Introduction}

Detonation is a self-sustained combustion driven wave that propagates at supersonic speed. It is different from deflagration which is the subsonic combustion propagating via diffusion mechanism. Due to their destructive nature, unintentional detonation phenomena are issues of high concern in process industries for safety assessment [1-4]. On the other hand, it is also because of its combustion properties that a controlled detonation with safe operation procedure has several benefits in practical engineering application, for example, in coating technology (e.g., [5-7]), as well as new propulsion system design particularly in recent decade the concept of pulse detonation engines (e.g., [8-10]) and microreactor devices (e.g., [11, 12]). Either for suppressing unintentional detonation or safely using its controlled propagation a thorough knowledge of the conditions under which a detonation can be favorably initiated and its propagation can be sustained is of interest for many engineering applications and industrial processes.

Detonation limit generally refers to the condition, outside of which the detonation wave fails to propagate [13]. Although the detonation limit can be defined in a number of ways and in fact depends on a combination of many factors, e.g., tube diameter, the initial thermodynamic state of the mixture and the mixture composition, etc., here in this study 'limit' is defined as the minimum tube diameter where the influence of boundary conditions leads to the onset of detonation limit and below which a detonation fails to propagate. From the engineering point-of-view, detonation limit is equivalent to the conventional safety parameter of quenching distance or maximum experimental safe gap (MESG) for deflagration used commonly for safety assessment [14], however the mechanism of failure is totally different due to various distinct physical processes that control the propagation. In any case, the detonation limit is particularly important to the design of detonation arrestors which is essentially an obstruction that is placed in the path of 


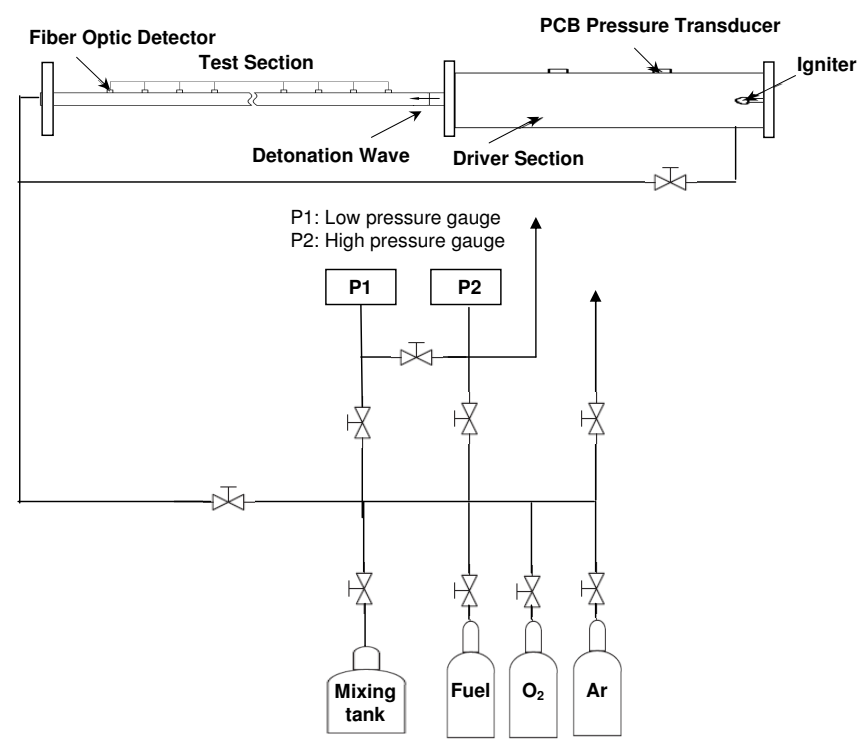

Fig. 1 A schematic of the experimental apparatus.

a detonation to quench the chemical reactions and the propagation of the detonation further downstream, for example in a pipeline [15]. It is also important in the design of detonation-based micro-thrusters to ensure that a detonation can propagate through the microchannel of different inner diameters; Similarly in microreactor devices safety requires the description of the extended range of operation conditions to know whether an explosion can propagate through the microchannel and cause ignition in a subsequent volume containing detonative materials [11].

In this study, new experiments were performed to investigate the limit phenomenon in long test tubes with a wide range of diameters (from $50.8 \mathrm{~mm}$ down to 1.5 $\mathrm{mm}$ in diameter) in a number of different stoichiometric fuel-oxygen-diluent mixtures. For the purpose of safety engineering, critical limits or the minimum tube diameters for the steady detonation propagation were determined from the experimental measurements in a number of hydrocarbon combustible mixtures. Using the present results, this study further establishes the applicability of the $\lambda / 3$ rule (where $\lambda$ denotes the detonation cell size) to estimate the minimum tube diameter for detonation propagation over an extended range in diameter scale $[2$, $13]$.

\section{Experimental details}

The detonation tube used in the present study consists of a steel driver section $65 \mathrm{~mm}$ diameter and $1.3 \mathrm{~m}$ long. The polycarbonate test tube of various diameters is attached to the end of the driver tube. Five different diameters, $D=1.5,3.2,12.7,31.7$ and $50.8 \mathrm{~mm}$, were used in the present study with total tube length $L=2438,2438$, 4118, 4118, $4118 \mathrm{~mm}$, respectively. Detonation was initi-

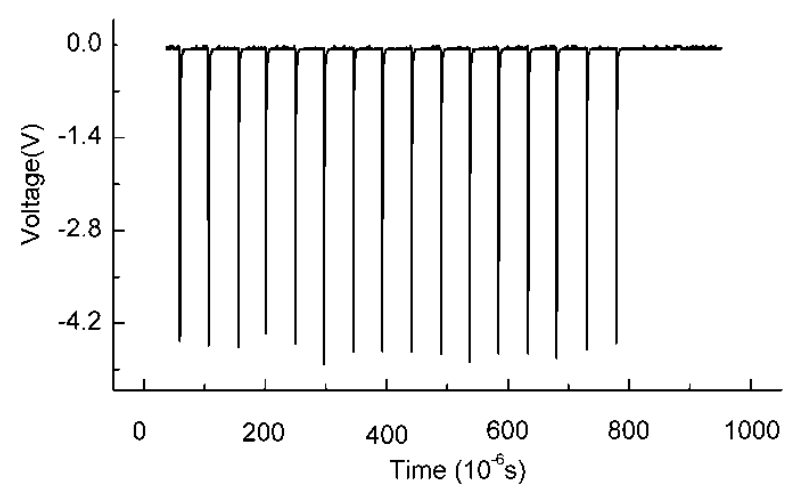

Fig. 2 Sample signals from the optical detectors.

ated by a high energy spark discharge and a short length of Shchelkin spiral was also inserted downstream of the spark plug to promote detonation initiation. For experiments with the small diameter tubes of $1.5 \mathrm{~mm}$ and $3.2 \mathrm{~mm}$ in diameter, a 1.5-long driver section filled with a more sensitive mixture (stoichiometric $\mathrm{C}_{2} \mathrm{H}_{2}+2.5 \mathrm{O}_{2}$ ) was used to facilitate detonation initiation and to ensure a Chapman-Jouguet (CJ) wave was formed prior to the detonation wave entering the test section. A schematic of the experimental apparatus and the flow sheet of the set-up are shown in Fig. 1.

Five explosives mixtures, i.e., $\mathrm{C}_{2} \mathrm{H}_{2}+2.5 \mathrm{O}_{2}+85 \% \mathrm{Ar}$, $\mathrm{C}_{2} \mathrm{H}_{2}+2.5 \mathrm{O}_{2}+70 \% \mathrm{Ar}, \mathrm{C}_{2} \mathrm{H}_{2}+5 \mathrm{~N}_{2} \mathrm{O}, \mathrm{C}_{3} \mathrm{H}_{8}+5 \mathrm{O}_{2}$, $\mathrm{CH}_{4}+2 \mathrm{O}_{2}$ were tested and studied. These fuel $/ \mathrm{O}_{2}$ compositions cover a wide range of detonation instability, from very stable ones with regular cellular patterns to highly unstable mixtures with irregular cellular detonation structures [16]. For safety engineering purposes, only the most explosive composition of each of these mixtures, i.e., the stochiometric condition, is investigated. The explosive mixtures of the desired composition were prepared via the partial pressure method and allowed to mix for at least 24 hours prior to being used. Piezoelectric transducers (PCB Piezotronics, Inc.) were used for pressure measurement and for the measurement of detonation velocity, $2 \mathrm{~mm}$ diameter fiber optics were spaced periodically along the entire length of the test section. Detonation velocity was determined from the time-ofarrival of the detonation at various optical probe locations. Typical output from the optical detectors is shown in Fig. 2.

\section{Results and discussion}

The variation of detonation velocity with initial pressure and tube diameter is first discussed. Figure 3 exemplarily shows the velocity measurement along the small tubes for the stoichiometric $\mathrm{C}_{3} \mathrm{H}_{8}+5 \mathrm{O}_{2}$ mixture. Practically, from the optical fibers measurement as shown in Fig. 2 , a local velocity (from two adjacent probes) can be computed. An overall averaged velocity over the length 

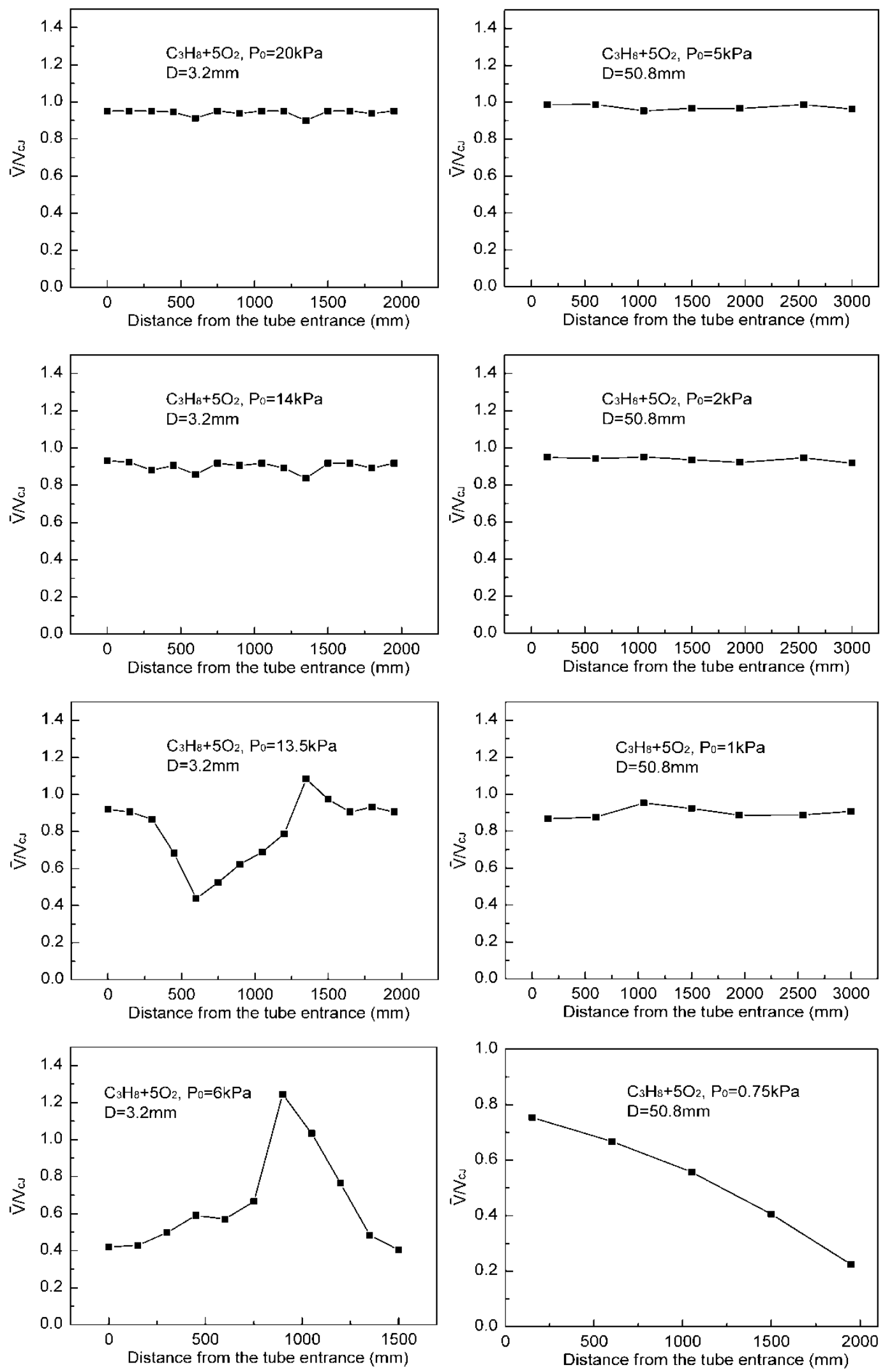

Fig. 3 Normalized propagation velocity inside the 3.2 and $50.8 \mathrm{~mm}$ diameter tubes with decreasing initial pressure for the $\mathrm{C}_{3} \mathrm{H}_{8}+5 \mathrm{O}_{2}$ mixture. 

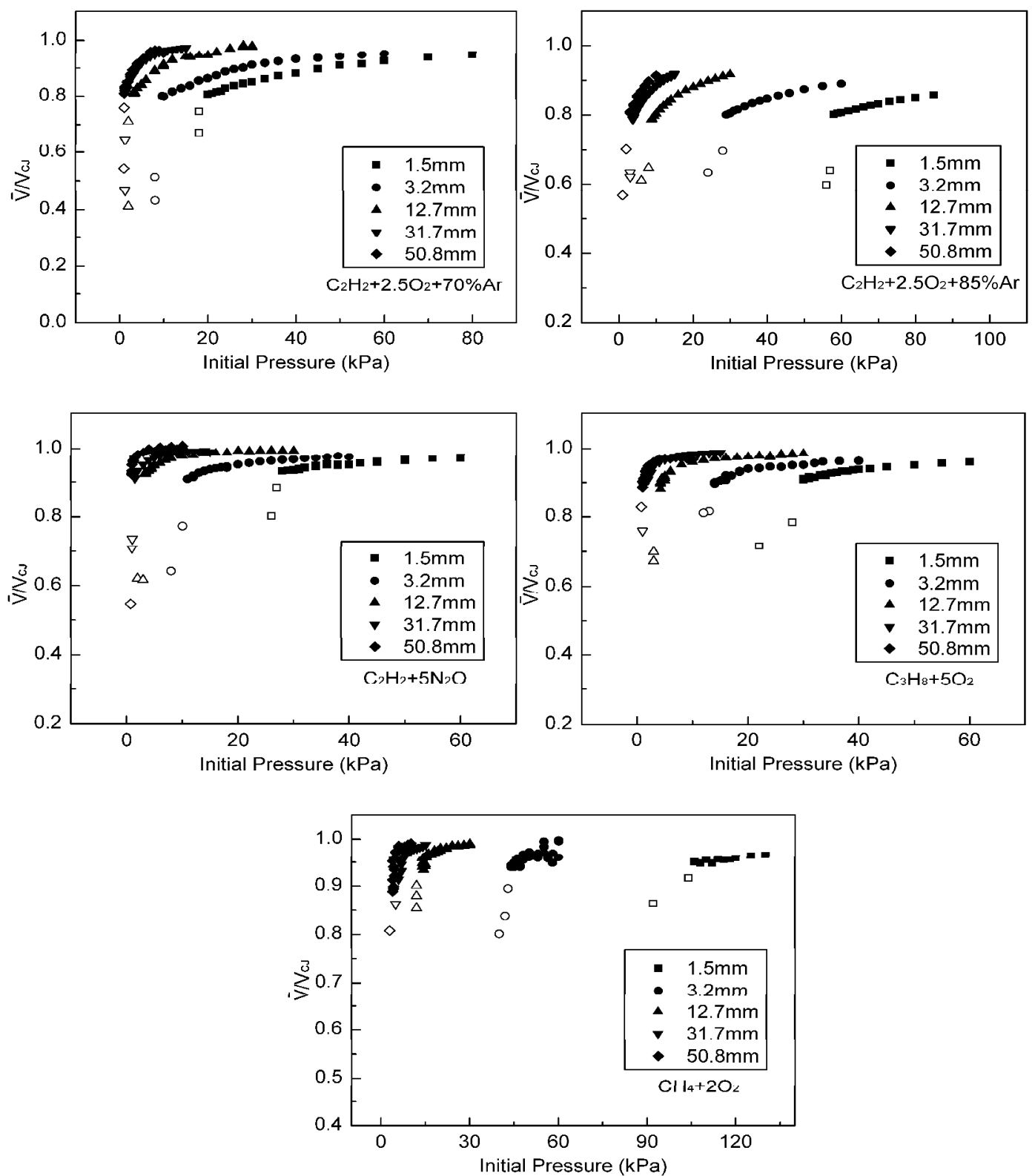

Fig. 4 Normalized propagation velocity as function of the initial pressure for different tube diameters and mixtures. (Open symbols are used to show the average values of the local propagation velocity fluctuations in the vicinity of the detonation limit.)

of the tube can also be deduced from Fig. 3. In general for all tested mixtures, at high initial pressures and large tube diameters (well within the limits), the propagation velocities approach the Chapman-Jouguet (CJ) velocity value computed using the chemical equilibrium software CEA [17]. The fluctuations in the local velocity are also small and an essentially steady velocity can be determined. By reducing the initial pressure, hence the sensitivity of the mixture, the onset of limit is approached and the velocity deficit increases. As observed in a wealth of other studies in the literature (e.g., [18$24]$ ), the onset of limit (or condition close to the limit), particularly for undiluted hydrocarbon mixtures, is signified by large velocity fluctuation showing a spectrum of different unstable behaviors. These unstable phenomena are more pronounced in small tube diameters where the boundary layer influences largely the wave propagation [23]. Close to failure, the fluctuation of the local velocity can be quite significant and the local velocity fluctuations are often used to only provide a qualitative idea of the near limit behavior [24]. In fact, these velocity fluctuations or unstable modes depend strongly on the specific boundary conditions and therefore, for practical purpose, it is thus not very useful to consider a global averaged 

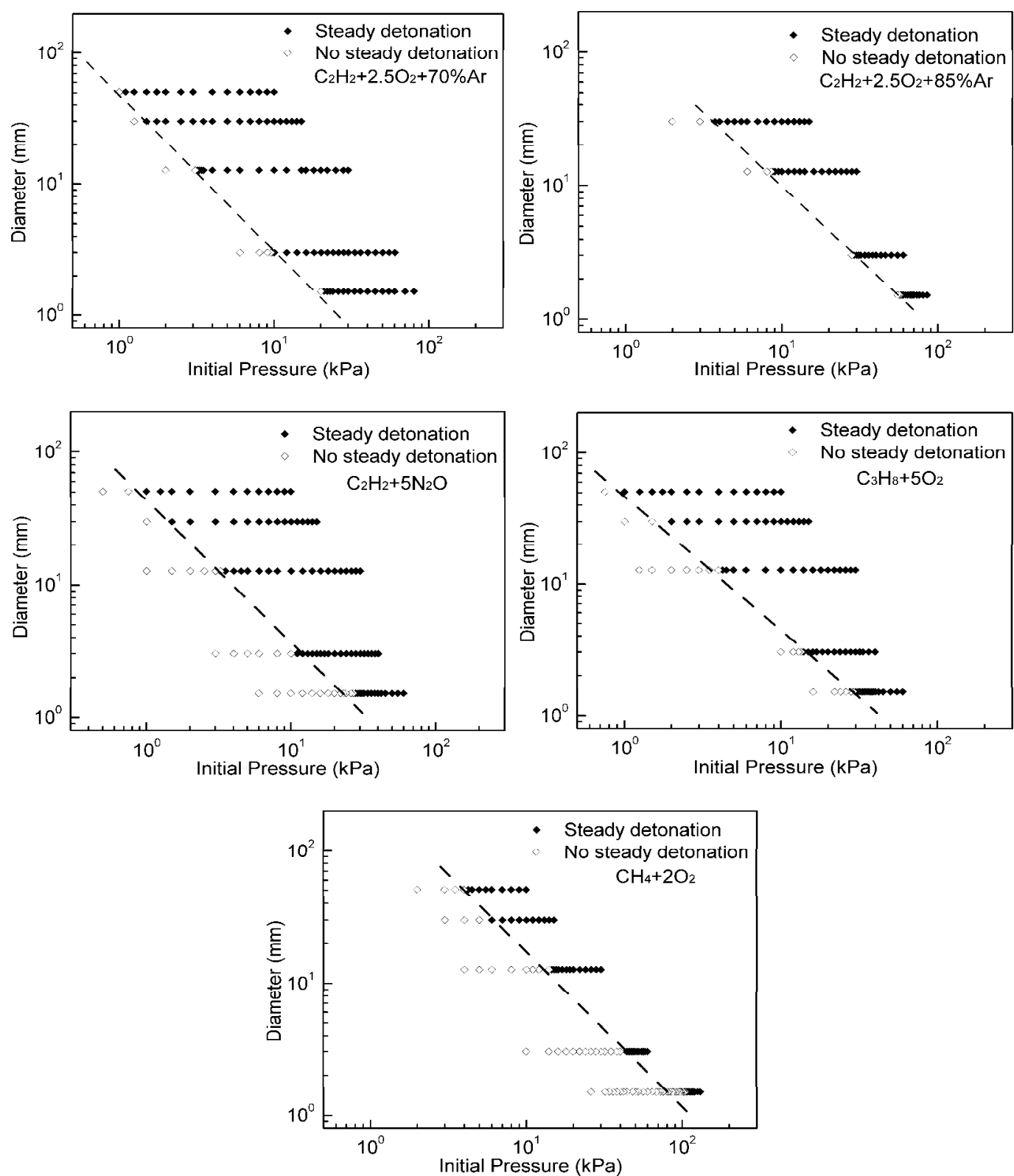

Fig. 5 Determination of minimum tube diameters as function of the initial pressure for all tested mixtures.

value of these local velocities to define a universal safety limit parameter. Furthermore, averaging any fluctuating quantity often ignores or hides some important physics and can often lead to some mis-interpretation of the phenomenon. Hence, the detonation limit is determined in this study as the critical condition past the existence of a steady minimum propagating velocity; and the onset of detonation limit is generally indicated by a continuous decay of this steady velocity until it can no longer be observed. In Fig. 4, the steady velocity normalized with the CJ value (or equivalently the velocity deficit) as a function of the initial pressure is plotted for different tubes and all tested mixtures. The filled black data points in the figure represent the measured steady ve- locity results. For comparison and clarity, the averaged velocities below the critical initial pressure for steady detonation propagation, i.e., average values of the local propagation velocity fluctuations due to various unstable behaviors in the vicinity of the detonation limit are also given using the open symbols in these plots. These average velocities indeed follow very well the trend of the velocity deficits from the steady velocity data. As the limit approaches, an abrupt drop in velocity deficit can be observed in all cases with the appearance of some velocity fluctuations. It is perhaps worth mentioning that in highly argon-diluted mixtures, past the critical pressure limit defined by the steady velocity criterion, the wave fails generally with a further slight decrease in ini- 


\begin{tabular}{ccc}
\hline Mixture & $A$ & $m$ \\
\hline $\mathrm{C}_{2} \mathrm{H}_{2}+2.5 \mathrm{O}_{2}+85 \% \mathrm{Ar}$ & 83.87 & 0.91 \\
$\mathrm{C}_{2} \mathrm{H}_{2}+2.5 \mathrm{O}_{2}+70 \% \mathrm{Ar}$ & 28.64 & 0.90 \\
$\mathrm{C}_{2} \mathrm{H}_{2}+5 \mathrm{~N}_{2} \mathrm{O}$ & 42.69 & 1.05 \\
$\mathrm{C}_{3} \mathrm{H}_{8}+5 \mathrm{O}_{2}$ & 44.23 & 0.97 \\
$\mathrm{CH}_{4}+2 \mathrm{O}_{2}$ & 161.88 & 1.07 \\
\hline
\end{tabular}

Table 1 Minimum tube diameter for detonation propagation as function of the initial pressure given by $D_{\min }[\mathrm{mm}]=$ $A\left(P_{o}[\mathrm{kPa}]\right)^{-m}$

tial pressure. However, for undiluted mixtures the results are observed to be different. Past the critical limit defined in this work using the results of steady velocity, detonations in undiluted mixtures can still exhibit different unstable propagation modes. Therefore, average values of the velocity fluctuations due to these unstable behaviors can still be estimated and plotted for a relatively large pressure range below the steady state velocity limit. Such difference can perhaps be explained by the mechanisms responsible for the failure in both argon-diluted and undiluted mixtures. As discussed in previous studies on detonation limits [23], detonations in highly argon-diluted mixtures are failed by the wave curvature caused by the boundary layer effect. Past the critical frontal curvature, a steady detonation can longer exist, leading to failure or limit. In contrast, at critical conditions instability at the detonation front plays a significant role in undiluted mixtures. Although the propagation may be unsteady with significant velocity fluctuation, the instability effect provides the detonation wave an ability to maintain its propagation and causes different near-limit unstable behaviors [13]. In general, it can also be seen from Fig. 4 that the smaller the tube diameter the higher is the critical pressure for limit, and the smaller is the propagation velocity at fixed initial pressure.

Using these present results, Fig. 5 summarizes alternatively the Go/NoGo plots as a function of the initial pressure for different diameter tubes. The corresponding limit (or the maximum safe diameter as named by Fischer et al., [11]) can be depicted more clearly in these $\log$-log diagrams versus the initial pressure. The powerlaw correlations between the minimum tube diameters for detonation propagation as a function of initial pressure for different mixtures obtained from least-square regression are presented in Table 1.

It is established that detonation usually propagates in a tube diameter with at least one-third the size of detonation cell width $\lambda$, the so-called the $\lambda / 3$ rule [2]. As explained by Lee [25], since $\pi \cdot D$ represents the largest characteristic length scale of the tube it should correlate with the length scale that characterizes the sensitivity of the mixture, i.e., detonation cell size $\lambda$. This argument thus leads to the $\lambda / 3$ rule. Here, it is thus worth to correlate the present minimum diameter results with available detonation cell size data mostly tabulated in the CAL-

\begin{tabular}{ccc}
\hline Mixture & $C$ & $n$ \\
\hline $\mathrm{C}_{2} \mathrm{H}_{2}+2.5 \mathrm{O}_{2}+70 \% \mathrm{Ar}$ & 348.1 & 1.34 \\
$\mathrm{C}_{2} \mathrm{H}_{2}+5 \mathrm{~N}_{2} \mathrm{O}$ & 115.6 & 1.13 \\
$\mathrm{C}_{3} \mathrm{H}_{8}+5 \mathrm{O}_{2}$ & 230.0 & 1.27 \\
$\mathrm{CH}_{4}+2 \mathrm{O}_{2}$ & 996.0 & 1.28
\end{tabular}

Table 2 Cell size correlations for mixtures as function of the initial pressure given by $\lambda[\mathrm{mm}]=C\left(P_{o}[\mathrm{kPa}]\right)^{-n}$. (Cell size for the $\mathrm{C}_{2} \mathrm{H}_{2}+5 \mathrm{~N}_{2} \mathrm{O}$ is determined using the universal critical tube diameter relationship $d_{c}=13 \lambda$ (Zhang et al. $[28]))$

TECH Detonation Database [26] to validate again this rule. The curve fit correlations of available cell size data as a function of initial pressure for the mixtures considered in this study (except $\mathrm{C}_{2} \mathrm{H}_{2}+2.5 \mathrm{O}_{2}+85 \% \mathrm{Ar}$ of which cell size data are not readily available) were derived previously by Radulescu [27], Zhang et al. [28] and Lee et al. [24]; and these are summarized in Table 2. Figure 6 also shows the $\lambda / 3$ corresponding to each limiting condition of each experiment, as well as the correlation from all experimental data represented by the dashed line. Figure 6 is presented in the same format as the data published by Fischer et al. [11] in order to present all key results in one single plot and to determine the limit. By comparing the minimum tube diameter $D$ for detonation propagation and cell size $\lambda$ at the limit condition, it is found that for all mixtures the boundary defining the limit is reasonably close to $D \sim \lambda / 3$ as shown in Fig. 6 . Therefore, the $\lambda / 3$ rule is valid and can be used for most practical purposes to determine the limit condition once the detonation cell width is known from either model estimation or interpolation of experimental data. For conservative consideration, due to the inherent uncertainty in the cell size measurement and the unstable phenomenon at the onset of limit which sometime render its definition difficult, it may be necessary to introduce a safety factor of 0.5 as suggested by Fischer et al. [11], i.e., $D \approx 0.5 \cdot \lambda / 3$ represented by the solid line in Fig. 6. This could in fact cover the deviation of smallest minimum diameters determined from the present experimental measurement.

\section{Concluding remarks}

In this paper, the limit of detonation propagation in round tubes is investigated. Five tube diameters with large $L / D$ ratio and five different combustible mixtures are considered for the experiments. Using the steady velocity deficit measurement to determine the limit, the dependency of minimum tube diameter for detonation propagation on initial pressure is obtained. These measured velocity data shall be useful for safety assessment in process industries and also in developing and validating models for detonation limits $[29,30]$. By comparing the detonation width with the minimum tube diameter 


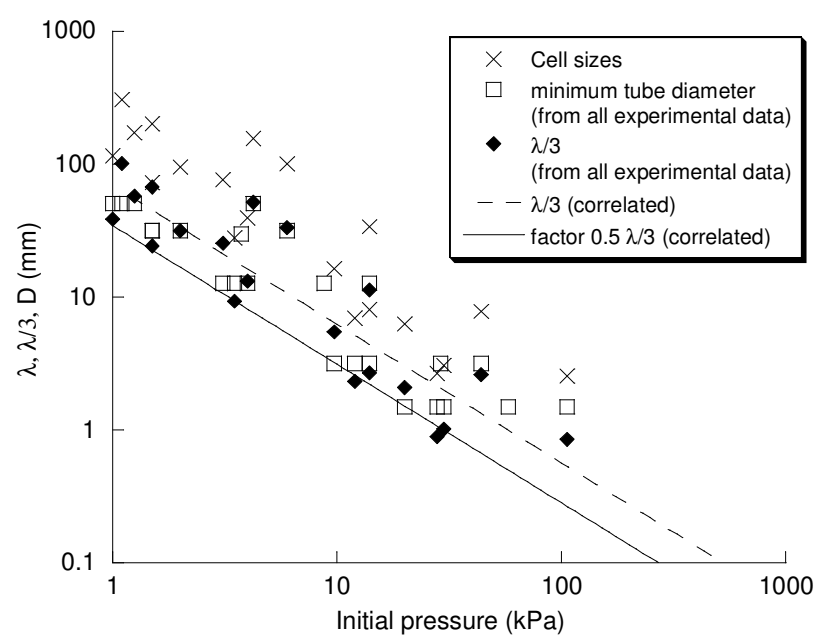

Fig. 6 Determination of the limit criterion and validation of the $\lambda / 3$ rule.

for detonation propagation, the $\lambda / 3$ rule is found to be applicable over a range of diameter tube size, and indeed should also be valid for micro-scale tube as found by Fischer et al. [11] and Brandes et al. [31]. Hence, for safety engineering purpose this criterion serves well as an appropriate estimation of minimum tube diameter for detonation propagation over a range of diameter scales. Nevertheless, to understand further the detonation limit and the physical mechanism responsible for this phenomenon in order to come up with a more fundamental criterion to define the minimum tube diameter for propagation, a closer look and analysis of the instability of the propagating cellular detonation front as the limits are approached is needed.

Acknowledgements This work is supported by the Natural Sciences and Engineering Research Council of Canada (NSERC).

\section{References}

1. Matsui, H. and Lee, J.H.S.: On the measure of the relative detonation hazards of gaseous fuel-oxygen and air mixtures. Proc. Combust. Inst. 17, 1269-1280 (1978).

2. Nettleton, M.A.: Gaseous Detonation: Their Nature, Effects and Control. Chapman \& Hall Ltd. London (1987).

3. Hirano, T.: Combustion science for safety. Proc. Combust. Inst. 29, 167-180 (2002).

4. Ng, H.D. and Lee, J.H.S.: Comments on explosion problems for hydrogen safety. J. Loss Prev. Process Ind. 21(2), 136-146 (2008).

5. Kharlamov, Y.A.: Detonation spraying of protective coatings. Materials Sci. Eng. 93, 1-37 (1987).

6. Tyurin, Yu. N. and Pogrebnjak, A.D.: Advances in the development of detonation technologies and equipment for coating deposition. Surf. Coat. Technol. 111(2-3), 269-275 (1999).

7. Singh, L., Chawla, V. and Grewal, J.S.: A review on detonation gun sprayed coatings. J. Minerals Materials Characterization and Engineering 11(3), 243-265 (2012).
8. Roy, G.D., Frolov, S.M., Borisov, A.A. and Netzer, D.W.: Pulse detonation propulsion: challenges, current status, and future perspective. Prog. Energy Combust. Sci. 30, 545-672 (2004)

9. Wu, M.H. and Lu, T.H.: Development of a chemical microthruster based on pulsed detonation. J. Micromech. Microeng. 22(10), 105040 (2012).

10. Phylippov, Yu. G., Dushin, V.R., Nikitin, V.F., Nerchenko, V.A., Korolkova, N.V. and Guendugov, V.M.: Fluid mechanics of pulse detonation thrusters. Acta Astronautica 76, 115-126 (2012).

11. Fischer, J., Liebner, C., Hieronymus, H. and Klemm, E.: Maximum safe diameters of microcapillaries for a stoichiometric ethene/oxygen mixture. Chem. Eng. Sci. 64, 29512956 (2009)

12. Heinrich, S., Edeling, F., Liebner, C., Hieronymus, H., Lange, T. and Klemm, E.: Catalyst as ignition source of an explosion inside a microreactor. Chem. Eng. Sci. 84 , 540-543 (2012).

13. Lee, J.H.S.: The Detonation Phenomenon. Cambridge University Press, New York (2008).

14. Britton, L.G.: Using maximum experimental safe gap to select flame arresters. Process Saf. Prog. 19(3), 140-145 (2000).

15. Thomas, G.O. and Teodorczyk, A.: An evaluation of new procedures for testing explosion arresters. Process Safety and Environmental Protection, 76(4), 277-285 (1998).

16. Ng, H.D. and Zhang, F.: Detonation Instability. In: Zhang, F. (Ed.), Shock Waves Science and Technology Library, Vol 6: Detonation Dynamics, Springer, Berlin Heidelberg, Chap. 3 (2012).

17. Gordon, S. and McBride, M.J.: Computer program for calculation of complex chemical equilibrium compositions and applications. RP-1311, NASA Reference Publication, Cleveland (1996).

18. Dupré, G., Joannon, J., Knystautas, R. and Lee, J.H.: Unstable detonations in the near-limit regime in tubes. Proc. Combust. Inst. 23, 18131820 (1990).

19. Lee, J.J., Dupré, G., Knystautas, R. and Lee, J.H.: Doppler interferometry study of unstable detonations. Shock Waves 5, 175-181 (1995).

20. Haloua, F., Brouillette, M., Lienhart, V. and Dupré, G.: Characteristics of unstable detonations near extinction limits. Combust. Flame 122(4), 422-438 (2000).

21. Kitano, S., Fukao, M., Susa, A., Tsuboi, N., Hayashi, A.K. and Koshi, M.: 2009: Spinning detonation and velocity deficit in small diameter tubes. Proc. Combust. Inst. 32(2), 2355-2362 (2009).

22. Jackson, S., Lee, B.J., Huang, W., Pintgen, F., Karnesky, J., Liang, Z. and Shepherd, J.E.: Experimental detonation propagation under high loss conditions. Proc. 22nd Int. Colloq. Dynamics Expl. Reac. Sys., Minsk, Belarus (2009).

23. Camargo, A., Ng, H.D., Chao, J. and Lee, J.H.S.: Propagation of near-limit gaseous detonations in small diameter tubes. Shock Waves 20(6), 499-508 (2010).

24. Lee, J.H.S., Jesuthasan, A. and Ng, H.D.: Near limit behavior of the detonation velocity. Proc. Combust. Inst. 34(2), 1957-1963 (2013).

25. Lee, J.H.S.: Dynamic Parameters of Gaseous Detonations. Ann. Rev. Fluid Mech. 16, 311-336 (1984).

26. Kaneshige, M. and Shepherd, J.E.: Detonation database, GALCIT Technical Report FM97-8, http://www.galcit.caltech.edu/detn_db/html/, 1997.

27. Radulescu, M.I.: The Propagation and Failure Mechanism of Gaseous Detonations: Experiments in PorousWalled Tubes. Ph.D. thesis, McGill University, Canada (2003).

28. Zhang, B., Ng, H.D. and Lee, J.H.S.: Dynamic detonation parameters in $\mathrm{C}_{2} \mathrm{H}_{2} / \mathrm{N}_{2} \mathrm{O} / \mathrm{Ar}$ mixtures. Combust. Flame 159(9), 2944-2953 (2012). 
29. Sadahira, K., Kitawaki, Y., Inaba, T., Susa, A., Matsuoka, K., Johzaki, T., and Endo, T.: Velocity deficits of $\mathrm{Ar}$ and $\mathrm{He}$ diluted $\mathrm{H}_{2}-\mathrm{O}_{2}$ system in small diameter tubes. In: Proc. 24th Int. Colloq. Dynamics Expl. Reac. Sys., July 28-Aug. 2, Taipei, Taiwan (2013)

30. Ishii, K. and Monwar, M.: Detonation propagation with velocity deficits in narrow channels. Proc. Combust. Inst. 33(2), 2359-2366 (2011)

31. Brandes, E., Gödde, M. and Hirsch, W.: Detonation parameters: a basis for the design of microstructured process equipment. Green Processing and Synthesis 1(4), 345-352 (2012). 